\title{
BMJ Open Does psychedelic drug use reduce risk of suicidality? Evidence from a longitudinal community-based cohort of marginalised women in a Canadian setting
}

\author{
Elena Argento, ${ }^{1,2}$ Steffanie A Strathdee ${ }^{3}$ Kenneth Tupper, ${ }^{4,5}$ Melissa Braschel, ${ }^{1}$ \\ Evan Wood, ${ }^{4,5}$ Kate Shannon ${ }^{1,4}$
}

To cite: Argento $\mathrm{E}$,

Strathdee SA, Tupper K, et al. Does psychedelic drug use reduce risk of suicidality? Evidence from a longitudinal community-based cohort of marginalised women in a Canadian setting. BMJ Open 2017;7:e016025. doi:10.1136/ bmjopen-2017-016025

- Prepublication history for this paper is available online. To view these files please visit the journal online (http://dx.doi org/10.1136/bmjopen-2017016025).

Received 23 January 2017

Revised 18 July 2017

Accepted 8 August 2017

CrossMark

${ }^{1}$ Gender and Sexual Health Initiative, St. Paul's Hospital, Vancouver, Canada

${ }^{2}$ Interdisciplinary Studies Graduate Program, University of British Columbia, Vancouver, Canada

${ }^{3}$ Department of Medicine, University of California San Diego, California, USA

${ }^{4}$ Faculty of Medicine, University of British Columbia, Vancouver, Canada

${ }^{5} \mathrm{BC}$ Centre on Substance Use, St. Paul's Hospital, Vancouver, Canada

Correspondence to Dr Kate Shannon; gshi@cfenet.ubc.ca

\section{ABSTRACT}

Objective This study aimed to longitudinally investigate whether ever having used a psychedelic drug can have a protective effect on incidence of suicidality among marginalised women.

Design Longitudinal community-based cohort study. Setting Data were drawn from a prospective, communitybased cohort of marginalised women in Metro Vancouver, Canada.

Participants 766 women completed the baseline questionnaire between January 2010 and August 2014. Participants who did not report suicidality at baseline and who completed at least one follow-up visit were included. Main outcome measure Extended Cox regression was used to model predictors of new suicidality (suicide ideation or attempts) over 54-month follow-up.

Results Nearly half ( $46 \% ; n=355)$ of participants reported prior suicidality and were thus excluded from the present analyses. Of 290 women eligible at baseline, $11 \%(n=31)$ reported recent suicidality during follow-up, with an incidence density of 4.42 per 100 person-years $(95 \% \mathrm{Cl}$ 3.10 to 6.30 ). In multivariable analysis, reported lifetime psychedelic drug use was associated with a $60 \%$ reduced hazard for suicidality (adjusted HR (AHR) 0.40; 95\% Cl 0.17 to 0.94). Crystal methamphetamine use (AHR 3.25; $95 \% \mathrm{Cl}$ 1.47 to 7.21 ) and childhood abuse (AHR 3.54; $95 \% \mathrm{Cl} 1.49$ to 8.40 ) remained independent predictors of suicidality. Conclusion The high rate of suicidality identified in this study is of major concern. Alongside emerging evidence on the potential of psychedelic-assisted therapy to treat some mental illness and addiction issues, our findings demonstrate that naturalistic psychedelic drug use is independently associated with reduced suicidality, while other illicit drug use and childhood trauma predispose women to suicidality. While observational, this study supports calls for further investigation of the therapeutic utility of psychedelic drugs in treating poor mental health and promoting mental wellness.

\section{INTRODUCTION}

Despite efforts to improve mental health over the last 60 years, suicide remains a critical

\section{Strengths and limitations of this study}

- To the best of our knowledge, this is the first study to longitudinally investigate the potential protective effect of psychedelic drug use on suicidality, drawing from a large, community-based cohort of marginalised women.

- Multivariable Cox regression analyses were used to examine the impact of lifetime psychedelic drug use on incidence of suicidality among a marginalised population.

- The associations between specific psychedelics, contexts of their use and suicidality were not explored in this study.

- Not all potential confounding variables could be controlled for in this study, and the associations uncovered cannot be determined as causal.

- Data were self-reported and variables examined included highly stigmatised topics, introducing the potential for recall bias, social desirability and reporting bias.

public health concern worldwide. ${ }^{12}$ Suicide was the second leading cause of death globally in 2012 among individuals aged $15-29$ years, ${ }^{2}$ with an estimated $80 \%-90 \%$ of suicide deaths attributable to mental health or substance use disorders. ${ }^{34}$ Significant gaps remain in empirical research examining suicidality among marginalised populations. Marginalised women, such as sex workers who are street involved or use drugs, experience disproportionately high levels of social and health-related risks and harms, including stigma, discrimination and violence ${ }^{5-7}$ as a result of dynamic structural drivers including poverty, criminalisation and racism. While sex workers are a diverse population working from indoor in-call and out-call venues to street-based settings, previous studies highlight substantial unmet mental health needs 
of more marginalised and street-involved sex workers. Studies among street-based sex workers and those who use drugs underscore the associations of social exclusion, depression and post-traumatic stress disorder (PTSD) with suicidality. ${ }^{8-12}$ Research demonstrates greater risk for suicidality among those with a history of trauma ${ }^{1314}$ and among street-involved sex workers who report historical experiences of violence and childhood abuse..$^{8-101516}$ Furthermore, indigenous women are vastly over-represented among street-based sex workers in North America and face devastating and multigenerational effects of trauma and socioeconomic dislocation (eg, high burden of mental illness and suicidality) as a result of colonialism, racialised policies and displacement from land and home communities. ${ }^{17} 18$

Various biological, interpersonal and sociostructural factors (eg, social exclusion/isolation, education level and employment) contribute to our understanding of suicidal behaviours. ${ }^{19}$ While evidence has demonstrated that some forms of cognitive behavioural therapy and pharmacological interventions may reduce suicidality, the literature is hampered by publication bias and significant heterogeneity of strategies and outcome measures. ${ }^{1420}$ Due to ethical challenges and limitations to studying suicide and its proxies (ie, ideation and attempts), there remains a paucity of evidence from randomised controlled trials to support the efficacy of prevention interventions. ${ }^{20}$ Researchers have largely focused on examining suicidality outcomes (rather than suicide itself), which may not be fully generalisable to understanding suicide or accurately evaluating treatment approaches. ${ }^{21}$ Furthermore, stigma continues to hinder research and reporting of suicidality. ${ }^{21}$ There remains an urgency to better understand pathways to suicidality, with literature highlighting the need for innovative psychological and psychosocial treatments ${ }^{14}$ and tailored intervention approaches for key marginalised populations. ${ }^{20} 21$ Given the complex aetiological pathways to suicide and limited effectiveness of well-established evidence-based interventions to reduce the burden of suicidality, the US National Institute of Mental Health has called for innovative research on suicide prevention and treatment for suicidality. $^{22}$

A number of psychedelic drug therapies are being revisited following a 40-year hiatus in research into their potential for the treatment of depression, anxiety, PTSD, eating disorders and addiction. ${ }^{23}$ Psychedelic drugs include the classic serotonergic psychedelics or 'hallucinogens' lysergic acid diethylamide (LSD), psilocybin, dimethyltryptamine and mescaline, as well as the 'enactogen' or 'empathogen' methylenedioxymethamphetamine (MDMA) ${ }^{23-25}$ all of which are being investigated in clinical/preclinical studies for their neuropharmacological functions and potential as adjuncts to psychotherapy. ${ }^{26-28}$ While renewed interest in psychedelic medicine is challenged by various funding and methodological and legal impediments, the emerging evidence indicating improved outcomes for some individuals suffering from mental health and addiction issues has generated new scientific inquiry and an imposing obligation to advance this research. ${ }^{23} 29{ }^{30}$ Recent observational studies in the USA demonstrate significant associations between lifetime psychedelic use and reduced recidivism and intimate partner violence among populations of prison inmates ${ }^{3132}$ and reduced psychological distress and suicidality among the general adult population. ${ }^{33-35}$

Despite the multifaceted structural and social inequities that shape poor mental health burden among marginalised and street-involved sex workers, there remains a paucity of data on suicide rates and research that systematically examines factors that potentiate or mitigate suicidality among sex workers, particularly in the global north. Some evidence suggests that psychedelic drug use may be protective with regard to suicidality ${ }^{33-35}$ and is associated with significant improvements in psychological well-being and reductions in depression and anxiety in clinical settings, ${ }^{36-41}$ yet existent research is characterised by large gaps. Given the urgency of addressing and preventing suicide and calls for prioritising innovative interventions, this study aimed to longitudinally investigate whether lifetime psychedelic drug use is associated with a reduced incidence of suicidality (suicide ideation or attempts) among a community-based prospective cohort of marginalised women. We postulated that psychedelic drug use would have an independent protective effect on suicidality over the study period.

\section{METHODS}

\section{Study design and participants}

Data for this study were drawn from a large, community-based, prospective cohort of women sex workers initiated in 2010, known as An Evaluation of Sex Workers Health Access (AESHA). Eligibility criteria for study participants included cisgender or transgender women, 14 years of age or older, who exchanged sex for money within the last 30 days. AESHA participants completed interviewer-administered questionnaires and HIV/sexually transmitted infection (STI)/hepatitis $\mathrm{C}$ virus (HCV) serology testing at enrolment and biannually. Experiential staff (current/former sex workers) are represented across interview, outreach and nursing teams, including coordinators with substantial community experience. Participants were recruited across Metro Vancouver using time-location sampling and community mapping strategies, with day and late-night outreach to outdoor sex work locations (ie, streets and alleyways), indoor sex work venues (ie, massage parlours, microbrothels and in-call locations) and online. Weekly outreach by experiential staff is conducted to over 100 sex work venues by outreach/nursing teams operating a mobile van, with regular contact as well as encouraging drop-in to womenonly spaces at the research office, contributing to an annual retention rate of $>90 \%$ for AESHA participants.

The main interview questionnaire elicits responses related to sociodemographics (eg, sexual and gender 
identities, ethnicity and housing), the work environment (eg, access to services, violence/safety, policing and incarceration), client characteristics (eg, types/fees of services and male condom use), intimate partners (eg, sexual history, cohabitation and financial support), trauma and violence (eg, lifetime and childhood trauma and exposure to intimate partner and workplace violence) and comprehensive injection and non-injection drug use patterns. The clinical questionnaire relates to overall physical, mental and emotional health, and HIV testing and treatment experiences to support education, referral and linkages with care. The research team works in close partnership with the affected community and a diversity of stakeholders (eg, legal/human rights experts, community-based organisations, service providers, health authorities, government officials and international policy bodies) and regularly engages in knowledge exchange efforts. AESHA is monitored by a Community Advisory Board of over 15 sex work, women's health and HIV service agencies, as well as representatives from the health authority and policy experts, and holds ethical approval through Providence Health Care/University of British Columbia Research Ethics Board. All participants receive an honorarium of \$C40 at each biannual visit for their time, expertise and travel.

To capture initial episodes of suicidality, analyses for this study were restricted to AESHA participants who had never thought about or attempted suicide at baseline and completed at least one follow-up visit between January 2010 and August 2014. Those with missing observations for suicidality at baseline $(n=50 / 766 ; 6.5 \%)$ were excluded from analysis, and one additional participant was excluded because reported suicidality was missing at follow-up.

\section{Statistical analyses}

The outcome of interest was a first episode of suicidality, defined as responding 'yes' to having thought about or attempted suicide in the last 6 months. Timefixed variables examined included age (continuous), gender/sexual minority (lesbian, gay, bisexual, trans or two-spirit), indigenous ancestry (inclusive of First Nations, Métis, and Inuit), being an immigrant/migrant worker (vs Canadian born), education (high school or greater) and physical and/or sexual childhood abuse (before age 18 years). Variables treated as time-updated covariates based on biannual follow-up data included HIV/STI serostatus, recent homelessness, recent physical and/or sexual violence by clients, recent police harassment and/or arrest and primary place to solicit clients in the last 6 months. Time-updated injection and non-injection drug use variables included lifetime use of psychedelics (including LSD/acid, magic mushrooms/ psilocybin, ecstasy/MDMA), cannabis, pharmaceutical opioids (any street methadone/suboxone, dilaudid, morphine, oxycontin, percocet/vicodin/demerol or T3s/T4s), crack, cocaine, crystal methamphetamine and heroin.
Using extended Cox regression, unadjusted hazard ratios (HR) and adjusted hazard ratios(AHR) and 95\% CI were calculated to identify predictors of suicidality. Psychedelic drug use, hypothesised a priori to be a predictor of suicidality, and variables that were significantly correlated with the outcome at the $\mathrm{p}<0.10$ level in bivariate analyses were subsequently fitted into a multivariable Cox model. Backward model selection was used to determine the final multivariable model with the best overall fit, as indicated by the lowest Akaike information criterion value. A complete case analysis was used, where observations with missing data were excluded from analyses, and participants who were lost to follow-up were right censored at their most recent study visit. All statistical analyses were performed using SAS software V.9.4. Two-sided $p$ values are reported.

\section{RESULTS}

\section{Sociodemographic characteristics}

Of the total 766 women who completed the baseline questionnaire, $46 \%(\mathrm{n}=355)$ reported ever experiencing suicidality (45\%; $\mathrm{n}=343$ reported suicidal thoughts, $32 \%$; $n=245$ attempted suicide) and were thus excluded from this analysis. A total of 290 women without suicidality who completed at least one follow-up visit were eligible for inclusion in the present analysis. Participants were followed for a total of 53.5 months (median=29.9 months). Baseline characteristics of participants who reported suicidality during follow-up compared with those who did not are displayed in table 1.

Overall, $11 \% \quad(\mathrm{n}=31)$ thought about or attempted suicide for the first time during follow-up, with an incidence density of 4.42 per 100 person-years (95\% CI 3.10 to 6.30$)$. The median age was 36 (IQR 29-42) and $16 \%$ $(\mathrm{n}=45)$ of participants identified as gender or sexual minorities. One-third $(\mathrm{n}=93)$ identified as indigenous and one-quarter $(n=73)$ had been homeless in the last 6 months. Half of the participants $(49 \% ; \mathrm{n}=142)$ solicited clients on the streets, and $37 \%(\mathrm{n}=106)$ solicited clients in indoor establishments. In terms of workplace violence and harassment, $14 \%(\mathrm{n}=41)$ and $37 \%(\mathrm{n}=107)$ reported recent exposure to physical and/or sexual violence from clients/dates and recent police harassment without arrest, respectively. Nearly half $(47 \% ; n=136)$ of participants reported ever being physically and/or sexually abused before age 18 years, and among those who reported suicidality, $77 \%$ ( $\mathrm{n}=24 / 31)$ experienced childhood abuse compared with $43 \%$ ( $n=112 / 259)$ among those who did not report suicidality $(\mathrm{p}<0.001)$.

\section{Substance use}

Over half of participants reported ever using crack $(63 \%, \mathrm{n}=184)$ or cocaine $(56 \% ; \mathrm{n}=162)$, with higher proportions of crack and cocaine use among those reporting suicidality than those who did not $(\mathrm{p}=0.003)$. Close to half $(49 \%, \mathrm{n}=141)$ reported lifetime heroin use, which was significantly higher among those who 
Table 1 Baseline characteristics of women in Vancouver who thought about or attempted suicide over follow-up, compared with those who did not $(\mathrm{n}=290)$

\begin{tabular}{|c|c|c|c|}
\hline Characteristics & Suicidality $n=31(11 \%)$ & No suicidality $n=259(89 \%)$ & p Value \\
\hline Age (median, IQR) & 34 (27 to 47$)$ & 36 (29 to 42$)$ & 0.767 \\
\hline Gender/sexual minority & $7(22.6)$ & $38(14.7)$ & 0.291 \\
\hline Born in Canada & 29 (93.6) & $156(60.2)$ & $<0.001$ \\
\hline Indigenous ancestry & $18(58.1)$ & $75(29.0)$ & 0.001 \\
\hline Education, high school or greater & $10(32.3)$ & $142(54.8)$ & 0.017 \\
\hline HIV seropositivity* & $7(22.6)$ & $26(10.0)$ & 0.065 \\
\hline Sexually transmitted infection seropositivity* & $3(9.7)$ & $35(13.5)$ & 0.778 \\
\hline Homelessness* & $10(32.3)$ & $63(24.3)$ & 0.336 \\
\hline Lifetime psychedelic use* & $8(25.8)$ & $71(27.4)$ & 0.849 \\
\hline Lifetime cannabis use* & $27(87.1)$ & $152(58.7)$ & 0.002 \\
\hline Lifetime prescription opioid use* & $12(38.7)$ & $77(29.7)$ & 0.306 \\
\hline Lifetime crack use* & $27(87.1)$ & $157(60.6)$ & 0.004 \\
\hline Lifetime cocaine use* & $25(80.7)$ & $137(52.9)$ & 0.003 \\
\hline Lifetime crystal methamphetamine use ${ }^{*}$ & $18(58.1)$ & $72(27.8)$ & $<0.001$ \\
\hline Lifetime heroin use ${ }^{*}$ & $21(67.7)$ & $120(46.3)$ & 0.024 \\
\hline Non-injection drug use ${ }^{\star}$ & $27(87.1)$ & $148(57.1)$ & 0.001 \\
\hline Injection drug use* & $16(51.6)$ & $81(31.3)$ & 0.023 \\
\hline Physical/sexual client violence* & $5(16.1)$ & $36(13.9)$ & 0.784 \\
\hline Physical/sexual childhood abuse & $24(77.4)$ & $112(43.2)$ & $<0.001$ \\
\hline Police harassment/arrest ${ }^{\star}$ & $16(51.6)$ & $91(35.1)$ & 0.072 \\
\hline \multicolumn{4}{|l|}{ Primary place to solicit clients* } \\
\hline Street/public space & $24(77.4)$ & $118(45.6)$ & \\
\hline Indoor/in-call venue & $3(9.7)$ & $103(39.8)$ & \\
\hline Independent/self-advertising (eg, newspapers and online) & $4(12.9)$ & $38(14.7)$ & 0.002 \\
\hline
\end{tabular}

*Time updated, last 6 months as reference.

reported suicidality than those who did not $(68 \%$ vs $46 \%, \mathrm{p}=0.02)$. Approximately one-third $(31 \%, \mathrm{n}=90)$ of participants had ever used crystal methamphetamine, and this was significantly higher among those who reported suicidality than those who did not $(58 \%$ vs $28 \%, \mathrm{p}<0.001)$. Overall, $27 \%(\mathrm{n}=79)$ of participants reported ever using a psychedelic substance, and of those, $75 \% \quad(n=59)$ had used MDMA/ecstasy, 35\% $(\mathrm{n}=28)$ had used LSD/acid and 30\% $(\mathrm{n}=24)$ had used psilocybin/magic mushrooms.

\section{Bivariate and multivariable Cox analyses}

Unadjusted HR and AHR for factors associated with a first episode of suicidality during follow-up are displayed in table 2. In the final multivariable model, crystal methamphetamine use (AHR 3.25; 95\% CI 1.47 to 7.21 ) and physical and/or sexual childhood abuse (AHR 3.54; $95 \%$ CI 1.49 to 8.40 ) remained independently associated with time to suicidality. Psychedelic use was associated with a $60 \%$ reduced hazard of suicidality (AHR 0.40; 95\% CI 0.17 to 0.94$)$.

\section{DISCUSSION}

This study demonstrated that among marginalised women, many of whom are street-involved and experience a disproportionate burden of violence, trauma, psychological distress and suicide, naturalistic psychedelic drug use predicted a significantly reduced hazard for suicidality. Crystal methamphetamine use and childhood abuse predisposed women to suicidality corresponding to more than a threefold increased hazard. Suicidality was highly prevalent, with almost half of women reporting lifetime suicidality at baseline, and $11 \%$ reporting a first episode of suicidality in the last 6 months during follow-up. Few studies have longitudinally examined predictors of suicidality among marginalised sex workers, and of the available data, most are cross-sectional and/or conducted in lower-income and middle-income settings. ${ }^{8} 164243$ The present study, based on a community-based, prospective cohort of marginalised women, adds to a growing body of literature documenting the protective and therapeutic potentials of psychedelic substances. ${ }^{23} 29394445$

Data were self-reported, and questions pertaining to events that occurred in the past may be subject to recall 
Table 2 Unadjusted and adjusted HRs for predictors of time to suicidality among women in Vancouver, 2010-2014 (n=290)

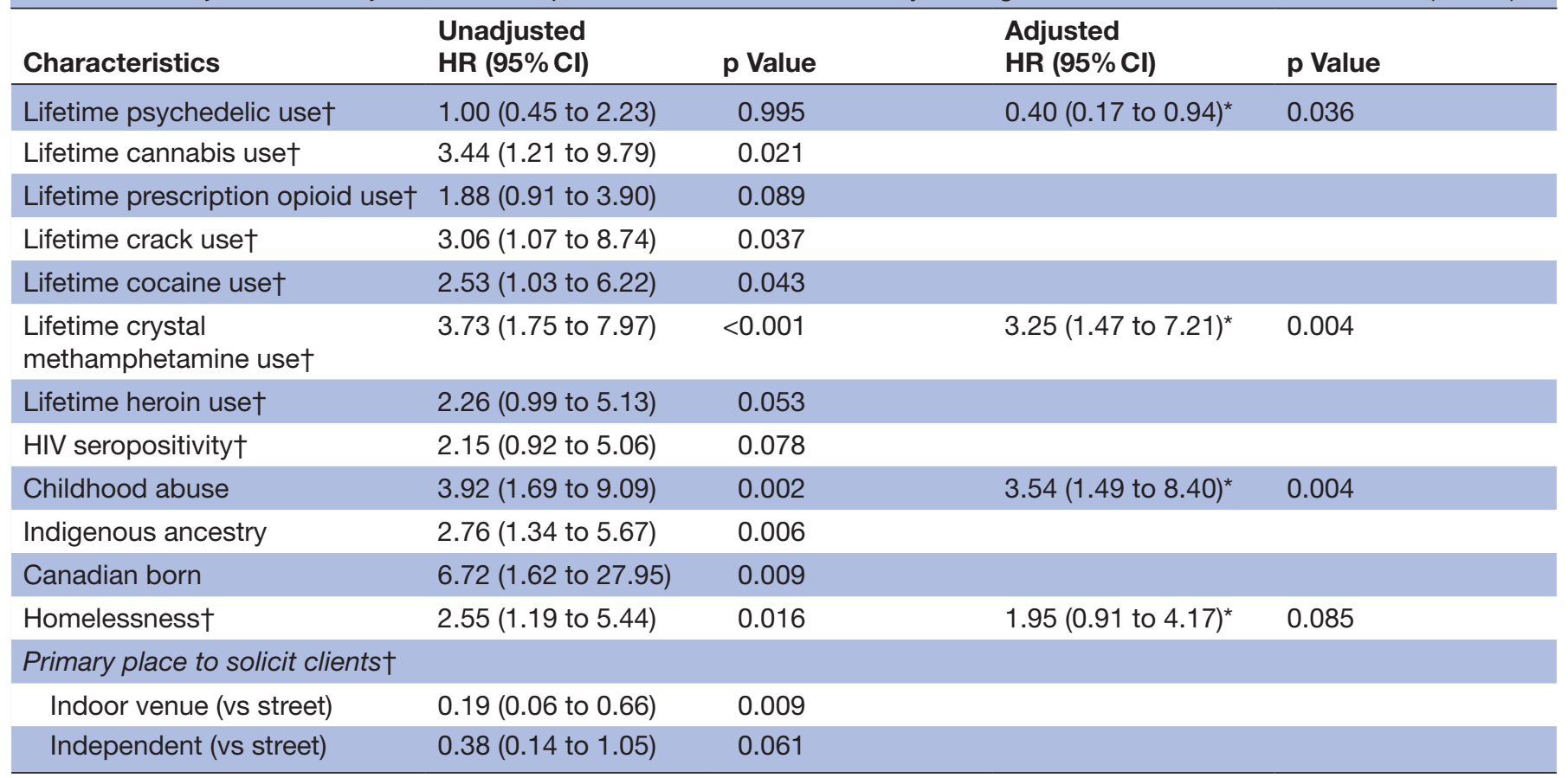

*Final multivariable model determined using backward selection with best overall fit, as indicated by the lowest Akaike information criterion value.

†Time updated, last 6 months as reference.

bias. Variables examined included sensitive and highly stigmatised topics such as childhood trauma, violence and illicit drug use, which introduce the potential for social desirability and reporting bias. However, the likelihood of these biases is reduced by the community-based nature of the study. While lifetime psychedelic drug use was found to reduce the hazard of suicidality, the associations uncovered in this analysis cannot be determined as causal. However, the use of Cox regression analysis in this study was able to determine a temporal relationship between psychedelic use and suicidality. The sample was restricted to participants who had not experienced suicidal ideation or attempt at baseline, ensuring that psychedelic use preceded suicidality and thus providing evidence that psychedelics have a protective effect. Due to a lack of statistical power, analyses evaluating the effects of more nuanced indicators of psychedelic use (eg, frequency of use or recent use), as well as separate analyses for ideation and attempt outcomes, were not feasible. Further examination of these variables would certainly be interesting and important in future analyses with additional data from follow-up questionnaires. Suicidality is influenced by complex individual, interpersonal and structural variables, and not all potential confounding variables could be controlled for in this study. For example, women who use psychedelics may also possess some characteristic(s) associated with a reduced likelihood of being suicidal (eg, openness to experience, curiosity or spirituality), which were not examined in this study. Despite the relative safety of psychedelic drug use as evidenced from the clinical and non-clinical literature, ${ }^{3646-49}$ it should be noted that the use of psychedelics, particularly with unknown doses sourced from unregulated street markets, is not without risk, highlighting the importance of set and setting ${ }^{23}$; the doses and contexts of psychedelic use among women in the present study could not be determined. The SE for the association between psychedelic use and suicidality was somewhat high, resulting in a wider CI. However, a large and significant protective effect was demonstrated in multivariable analysis, despite the relatively small number of events for suicidality over follow-up. With a larger sample size, we would expect a narrower CI for this association. The study population included women from a wide-ranging representation of sex work environments, yet findings may not be fully generalisable to sex workers in other settings. The mapping of working areas and time-location sampling helped to ensure a representative sample and to minimise selection bias.

To the best of our knowledge, this study is the first to longitudinally investigate associations with suicidality among marginalised and street-involved sex workers in North America and builds on prior cross-sectional research highlighting significantly elevated rates of suicidality and unmet mental health needs in this population. For example, a study conducted in Sydney, Australia demonstrated significant links between depression, trauma, and suicidality, where an estimated $42 \%$ of street-based female sex workers reported attempting suicide and $74 \%$ reported lifetime suicidal ideation. ${ }^{1042}$ While estimates of mental illness vary significantly across sex work settings, up to three-quarters of street-involved and drug-involved sex workers in a US study reported severe depression, 
anxiety or PTSD. ${ }^{12}$ Notably, our study demonstrated a lower risk of suicidality among women working indoors in bivariate analysis (HR 0.19, $\mathrm{p}=0.009$ ), lending support to the critical role of safer workplace environments in mitigating risk. In studies conducted in Asia, recent suicide attempts ranged from 19\% among sex workers in Goa, India ${ }^{16}$ to $38 \%$ among sex workers in China, ${ }^{81150}$ many of whom work in marginalised settings with few workplace protections. Transgender women involved in sex work, a subpopulation experiencing significant psychosocial vulnerability and discrimination, report notably further elevated rates of suicidality: three-quarters of participants in San Francisco reported suicide ideation, of whom 64\% attempted suicide. ${ }^{51}$ The global evidence is unequivocal that in settings where sex work is criminalised, sex workers are unable to access essential social, health and legal protections (eg, against violence), highlighting the need for structural (eg, decriminalisation) and community-led interventions to improve health and human rights. ${ }^{5}$ A structural approach to mitigating suicidality risk requires a reform of laws and policies that perpetuate stigma, discrimination, violence and unequal access to health and social supports among sex workers. Increased support for community-driven interventions that are gender and culturally appropriate are urgently needed, and any clinical treatment utilising psychedelics must be developed alongside sex worker-led interventions and community empowerment.

Our findings extend on research on associations between lifetime use of illicit drugs and increased risk for suicidality: in bivariate analysis, all classes of illicit drugs were demonstrated to increase the hazard of suicidality with the exception of psychedelics. In multivariable analysis, psychedelics were independently associated with a $60 \%$ reduced hazard for suicidality, contributing to emergent evidence on the potential of psychedelics to mitigate risks for suicide.

Among the various scientific studies examining the potential benefits of psychedelic drug use, a recent and large $(n>190000)$ population study conducted among adult respondents in the USA demonstrated that psychedelics are associated with reduced psychological distress and suicidality. ${ }^{33} \mathrm{~A}$ recent open-label trial conducted in the UK demonstrated the safety and efficacy of psilocybin for treating major depression, ${ }^{36}$ and another open-label trial in Brazil found rapid and sustained antidepressant effects from the Amazonian psychedelic brew ayahuasca administered in a clinical setting. ${ }^{38}$ The ways in which psychedelics may alleviate suffering associated with some mental illness is undoubtedly a complex phenomenon. It has been hypothesised that psychedelics modify neurobiological processes that may be involved in suicidality by downregulating $5-\mathrm{HT}_{2 \mathrm{~A}}$ serotonin receptors, as increased binding (and upregulation) of this receptor has been implicated in major depression and suicide. ${ }^{39} 4552$ Furthermore, there is evidence that psychedelics alter neural network connectivity and enhance recall of autobiographical memories, which may facilitate positive reprocessing of trauma. ${ }^{265354}$ Recent randomised, placebo-controlled, crossover studies found that psilocybin (among $n=25$ adults) and LSD (among $\mathrm{n}=20$ adults) were associated with increased positive mood and psychological well-being, ${ }^{3739}$ supporting other work demonstrating the antidepressive/ anxiolytic effects of psychedelics. ${ }^{27} 404655$ The potential of psychedelics to elicit 'mystical-type' experiences, with profound and sustained positive changes in attitudes and mood, may play a key role in addiction treatment interventions. ${ }^{41} 455657$ For example, psilocybin-assisted psychotherapy demonstrated high success in smoking cessation outcomes at 6 months follow-up (abstinence rates of $80 \%$ ), and mystical experiences generated from the psilocybin sessions were significantly correlated with elevated ratings of personal meaningfulness, well-being and life satisfaction. ${ }^{58}$ Randomised control trials in the USA and Switzerland have demonstrated significant longterm improvements among patients with treatment-resistant PTSD following MDMA-assisted psychotherapy, ${ }^{28} 59$ and further research is continuing in an international multisite phase $\mathrm{t} 3$ clinical trial.

Marginalised and street-based sex workers experience complex and synergistic effects between trauma, lack of workplace safety and mental health/substance use comorbidities that elevate risk of suicidality. Marginalised women and sex workers who use drugs report high rates of childhood abuse, ${ }^{60-62}$ which is associated with an increased likelihood of experiencing subsequent physical or sexual violence, as well as initiating injection drug use. ${ }^{63-65}$ For those suffering from emotional trauma stemming from violence, including indirect violence (ie, witnessing violence), there may be a proclivity to use drugs for self-medication. ${ }^{66}{ }^{67}$ Violence and sexual coercion have been found to be significantly associated with suicidality among sex worker populations in China and India. ${ }^{8} 1668$ As demonstrated in this study, having an early traumatic life event is a key risk factor for suicide among sex workers, a high proportion of whom are Indigenous, and experiencing historical trauma can have harmful intergenerational impacts. ${ }^{17}$ Given that historical experiences of violence and trauma denote significant risk for suicide, there is an urgent need to provide integrated, trauma-informed intervention services for sex workers and other marginalised populations. Currently available interventions and pharmacological treatments for suicidality show limited efficacy, and concerted efforts should be made to increase access to evidence-based treatments and to explore alternative approaches to improving mental health and well-being. Emerging research and evidence show positive outcomes with psychedelic-assisted treatments, which have demonstrated an excellent record of safety with few to no serious adverse effects reported. ${ }^{1}$ This study suggests psychedelic substances may hold promise as useful tools in addressing mental health issues and remediating risks for psychological distress and suicide. 


\section{CONCLUSION}

The high rate of suicidality among marginalised women identified in this study is a critical public health concern. In the context of emerging research and evidence on the therapeutic potential of psychedelics to treat mental health issues, our findings demonstrated that naturalistic psychedelic use was independently associated with reduced suicidality among sex workers, while other drug use and childhood trauma increased the hazard for suicidality. To the best of our knowledge, this is the first study to longitudinally investigate the relationship between psychedelic drug use and suicidality. While observational, this study supports calls for further investigation of the therapeutic utility of psychedelic drugs in treating mental illness and promoting mental wellness.

Acknowledgements We thank all those who contributed their time and expertise to this project, particularly participants, AESHA community advisory board members and partner agencies, and the AESHA team, including: Sarah Moreheart, Jennifer Morris, Sylvia Machat, Jane Li, Minshu Mo, Sherry Wu, Sylvia Machat, Emily Leake, Anita Dhanoa, Meaghan Thumath, Alka Murphy, Jenn McDermid, Tave Cole and Jaime Adams. We also thank Abby Rolston, Peter Vann, Erin Seatter and Patricia McDonald for their research and administrative support

Contributors EA and KS conceptualised and designed the study. EA, SAS, MB and KS contributed to the interpretation and analysis of data. EA wrote the first draft of the manuscript. SAS, KT, MB, EW and KS contributed to reviewing and editing the manuscript. All authors critically revised the manuscript and approved the final draft. KS is the guarantor.

Funding This study was supported by the US National Institutes of Health (R01DA028648) and MacAIDS. KS is partially supported by a Canada Research Chair in Global Sexual Health and HIV/AIDS and the Michael Smith Foundation for Health Research. EA is supported by a Canadian Institutes of Health Research Doctoral Award. SAS is partially supported by a NIDA merit award (R37DA019829). EW is supported in part by a Tier 1 Canada Research Chair in Inner-City Medicine award. The study funders had no role in the study design, data collection, analysis, interpretation, writing of the report or decision to submit the paper for publication.

Competing interests All authors have completed the ICMJE uniform disclosure format www.icmje.org/coi_disclosure.pdf (available on request from the corresponding author) and declare: no support from any organisation for the submitted work; no financial relationships with any organisations that might have an interest in the submitted work in the previous 3 years; no other relationships or activities that could appear to have influenced the submitted work.

Ethics approval This study holds ethical approval through Providence Health Care/ University of British Columbia Research Ethics Board.

Provenance and peer review Not commissioned; externally peer reviewed. Data sharing statement № additional data are available.

Open Access This is an Open Access article distributed in accordance with the Creative Commons Attribution Non Commercial (CC BY-NC 4.0) license, which permits others to distribute, remix, adapt, build upon this work non-commercially, and license their derivative works on different terms, provided the original work is properly cited and the use is non-commercial. See: http://creativecommons.org/ licenses/by-nc/4.0/

(c) Article author(s) (or their employer(s) unless otherwise stated in the text of the article) 2017. All rights reserved. No commercial use is permitted unless otherwise expressly granted.

\section{REFERENCES}

1. Hawton K, van Heeringen K. Suicide. Lancet 2009;373:1372-81.

2. World Health Organization. Preventing Suicide: A Global Imperative. Geneva, Switzerland 2014 http://www.who.int/mental_health/suicideprevention/world_report_2014/en/ (accessed Dec 2016).

3. Whiteford HA, Degenhardt L, Rehm J, et al. Global burden of disease attributable to mental and substance use disorders: findings from the Global Burden of Disease Study 2010. The Lancet 2013;382:1575-86.

4. Canada S. Suicide Rates: An Overview. Ottawa, 2015. http:// www.statcan.gc.ca/pub/82-624-x/2012001/article/11696-eng.htm (accessed Dec 2016).

5. Shannon K, Strathdee SA, Goldenberg SM, et al. Global epidemiology of HIV among female sex workers: influence of structural determinants. Lancet 2015;385:55-71.

6. Argento E, Muldoon KA, Duff P, et al. High prevalence and partner correlates of physical and sexual violence by intimate partners among street and off-street sex workers. PLoS One 2014;9:e102129.

7. Deering K, Amin A, Shoveller J, et al. A systematic review of the global magnitude and drivers of violence against sex workers. Am J Public Health 2014;104:e42-54.

8. Gu J, Lau JT, Li M, et al. Socio-ecological factors associated with depression, suicidal ideation and suicidal attempt among female injection drug users who are sex workers in China. Drug Alcohol Depend 2014;144:102-10.

9. Zhang L, Li X, Wang B, et al. Violence, stigma and mental health among female sex workers in China: A structural equation modeling. Women Health 2017;57:685-704.

10. Roxburgh A, Degenhardt L, Copeland J. Posttraumatic stress disorder among female street-based sex workers in the greater Sydney area, Australia. BMC Psychiatry 2006;6:1-12.

11. Jtf L, Tsui HY, Spy H, et al. Prevalence of psychological problems and relationships with condom use and HIV prevention behaviors among Chinese female sex workers in Hong Kong. AIDS Care 2010;22:659-68.

12. Surratt HL, Kurtz SP, Chen M, et al. HIV risk among female sex workers in Miami: the impact of violent victimization and untreated mental illness. AIDS Care 2012;24:553-61.

13. Hadland SE, Marshall BDL, Kerr T, et al. Suicide and history of childhood trauma among street youth. J Affect Disord 2012;136:377-80.

14. O'Connor RC, Nock MK. The psychology of suicidal behaviour. Lancet Psychiatry 2014;1:73-85.

15. Gilchrist G, Gruer L, Atkinson J. Comparison of drug use and psychiatric morbidity between prostitute and non-prostitute female drug users in Glasgow, Scotland. Addict Behav 2005;30:1019-23.

16. Shahmanesh M, Wayal S, Cowan F, et al. Suicidal Behavior Among Female Sex Workers in Goa, India: The Silent Epidemic. Am J Public Health 2009;99:1239-46.

17. Bingham B, Leo D, Zhang R, et al. Generational sex work and HIV risk among Indigenous women in a street-based urban Canadian setting. Cult Health Sex 2014;16:440-52.

18. Benoit C, Carroll D, Chaudhry M. In search of a healing place: Aboriginal women in Vancouver's Downtown Eastside. Soc Sci Med 2003;56:821-33.

19. Li Z, Page A, Martin G, et al. Attributable risk of psychiatric and socio-economic factors for suicide from individual-level, populationbased studies: a systematic review. Soc Sci Med 2011;72:608-16.

20. Zalsman G, Hawton K, Wasserman D, et al. Suicide prevention strategies revisited: 10-year systematic review. Lancet Psychiatry 2016;3:646-59.

21. Klonsky ED, May AM, Saffer BY, et al. Suicide, Suicide Attempts, and Suicidal Ideation. Annu Rev Clin Psychol 2016;12:307-30.

22. National Action Alliance for Suicide Prevention: Research Prioritization Task Force. 2014. A Prioritized Research Agenda for Suicide Prevention: An Action Plan to Save Lives. Rockville. www. suicide-research-agenda.org accessed Dec 2016.

23. Tupper KW, Wood E, Yensen R, et al. Psychedelic medicine: a reemerging therapeutic paradigm. CMAJ 2015;187:1054-9.

24. Nichols DE. Hallucinogens. Pharmacol Ther 2004;101:131-81.

25. Bedi G, Hyman D, de Wit H. Is Ecstasy an "Empathogen"? Effects of $\pm 3,4$-Methylenedioxymethamphetamine on Prosocial Feelings and Identification of Emotional States in Others. Biol Psychiatry 2010;68:1134-40.

26. Carhart-Harris RL, Leech R, Williams TM, et al. Implications for psychedelic-assisted psychotherapy: functional magnetic resonance imaging study with psilocybin. Br J Psychiatry 2012;200:238-44.

27. Dos Santos RG, Osório FL, Crippa JA, et al. Antidepressive, anxiolytic, and antiaddictive effects of ayahuasca, psilocybin and Iysergic acid diethylamide (LSD): a systematic review of clinical trials published in the last 25 years. Ther Adv Psychopharmacol 2016:6:193-213.

28. Oehen $\mathrm{P}$, Traber $\mathrm{R}$, Widmer $\mathrm{V}$, et al. A randomized, controlled pilot study of MDMA ( $\pm 3,4-$ Methylenedioxymethamphetamine)-assisted psychotherapy for treatment of resistant, chronic Post-Traumatic Stress Disorder (PTSD). J Psychopharmacol 2013;27:40-52.

29. Sessa B. Shaping the renaissance of psychedelic research. Lancet 2012;380:200-1. 
30. Sessa B, Johnson MW. Can psychedelic compounds play a part in drug dependence therapy? Br J Psychiatry 2015;206:1-3.

31. Hendricks PS, Clark CB, Johnson MW, et al. Hallucinogen use predicts reduced recidivism among substance-involved offenders under community corrections supervision. J Psychopharmacol 2014;28:62-6.

32. Walsh Z, Hendricks PS, Smith $\mathrm{S}$, et al. Hallucinogen use and intimate partner violence: Prospective evidence consistent with protective eff ects among men with histories of problematic substance use. J Psychopharmacol 2016;30:601-7.

33. Hendricks PS, Thorne CB, Clark CB, et al. Classic psychedelic use is associated with reduced psychological distress and suicidality in the United States adult population. J Psychopharmacol 2015;29:280-8.

34. Krebs TS, Johansen PØ. Psychedelics and mental health: a population study. PLoS One 2013;8:e63972.

35. $P-\varnothing \mathrm{J}$, Krebs TS. Psychedelics not linked to mental health problems or suicidal behavior: A population study. J Psychopharmacol 2015;29:270-9.

36. Carhart-Harris RL, Bolstridge M, Rucker J, et al. Psilocybin with psychological support for treatment-resistant depression: an openlabel feasibility study. Lancet Psychiatry 2016;3:619-27.

37. Carhart-Harris R, Kaelen M, Bolstridge M, et al. The paradoxical psychological effects of lysergic acid diethylamide (LSD). Psychol Med 2016;12:1.

38. Sanches RF, de Lima Osório F, Dos Santos RG, et al. Antidepressant Effects of a Single Dose of Ayahuasca in Patients With Recurrent Depression: A SPECT Study. J Clin Psychopharmacol 2016;36:77-81.

39. Kraehenmann R, Preller KH, Scheidegger M, et al. Psilocybininduced decrease in amygdala reactivity correlates with enhanced positive mood in healthy volunteers. Biol Psychiatry 2015;78:572-81.

40. Grob CS, Danforth AL, Chopra GS, et al. Pilot study of psilocybin treatment for anxiety in patients with advanced-stage cancer. Arch Gen Psychiatry 2011:68:71-8.

41. Griffiths RR, Johnson MW, Richards WA, et al. Psilocybin occasioned mystical-type experiences: immediate and persisting dose-related effects. Psychopharmacology 2011;218:649-65.

42. Roxburgh A, Degenhardt L, Copeland J, et al. Drug Dependence and Associated Risks Among Female Street-Based Sex Workers in the Greater Sydney Area, Australia. Subst Use Misuse 2008;43:1202-17.

43. Hong Y, Zhang C, Li X, et al. Partner violence and psychosocial distress among female sex workers in China. PLoS One 2013;8:e62290.

44. Dyck E. LSD: a new treatment emerging from the past. CMAJ 2015;187:1079-80

45. Bogenschutz MP, Johnson MW. Classic hallucinogens in the tr eatment of addictions. Prog Neuropsychopharmacol Biol Psychiatry 2016;64:250-8

46. Gasser P, Holstein D, Michel Y, et al. Safety and efficacy of lysergic acid diethylamide-assisted psychotherapy for anxiety associated with life-threatening diseases. J Nerv Ment Dis 2014;202:513-20.

47. Mithoefer MC, Wagner MT, Mithoefer AT, et al. The safety and efficacy of $\{+/-\} 3,4$-methylenedioxymethamphetamine-assisted psychotherapy in subjects with chronic, treatment-resistant posttraumatic stress disorder: the first randomized controlled pilot study. J Psychopharmacol 2011;25:439-52.

48. Winstock A, Barratt M, Ferris J, et al. Global Drug Survey. London, 2017. https://www.globaldrugsurvey.com/wp-content/themes/ globaldrugsurvey/results/GDS2017_key-findings-report_final.pdf (accessed June 2017).

49. Nutt DJ, King LA, Phillips LD. Drug harms in the UK: a multicriteria decision analysis. Lancet 2010;376:1558-65.

50. Jung M. Associations of physical and sexual health with suicide attempts among female sex workers in South Korea. Sex Disabil 2013;31:275-86.

51. Nemoto T, Bödeker B, Iwamoto M. Social support, exposure to violence and transphobia, and correlates of depression among male- to-female transgender women with a history of sex work. Am J Public Health 2011;101:1980-8.

52. Anisman H, Du L, Palkovits M, et al. Serotonin receptor subtype and p11 mRNA expression in stress-relevant brain regions of suicide and control subjects. J Psychiatry Neurosci 2008;33:131-41.

53. Carhart-Harris RL, Leech R, Hellyer PJ, et al. The entropic brain: a theory of conscious states informed by neuroimaging research with psychedelic drugs. 8, 2014.

54. Carhart-Harris RL, Erritzoe D, Williams T, et al. Neural correlates of the psychedelic state as determined by fMRI studies with psilocybin. Proc Natl Acad Sci U S A 2012;109:2138-43.

55. Osório FL, Sanches RF, Macedo LR, et al. Antidepressant effects of a single dose of ayahuasca in patients with recurrent depression: a preliminary report. Rev Bras Psiquiatr 2015:37:13-20.

56. Griffiths RR, Richards WA, McCann U, et al. Psilocybin can occasion mystical-type experiences having substantial and sustained personal meaning and spiritual significance. Psychopharmacology 2006;187:268-83.

57. Bouso JC, Palhano-Fontes F, Rodríguez-Fornells A, et al. Long-term use of psychedelic drugs is associated with differences in brain structure and personality in humans. Eur Neuropsychopharmacol 2015;25:483-92.

58. Johnson MW, Garcia-Romeu A, Cosimano MP, et al. Pilot study of the 5-HT2AR agonist psilocybin in the treatment of tobacco addiction. J Psychopharmacol 2014;28:983-92.

59. Mithoefer MC, Wagner MT, Mithoefer AT, et al. Durability of improvement in post-traumatic stress disorder symptoms and absence of harmful effects or drug dependency after 3,4-methylenedioxymethamphetamine-assisted psychotherapy: a prospective long-te rm follow-up study. J Psychopharmacol 2013;27:28-39.

60. Rio Navarro J, Cohen J, Rocillo Arechaga E, et al. Physical and sexual violence, mental health indicators, and treatment seeking among street-based population groups in Tegucigalpa, Honduras. Revista Panamericana de Salud Pública 2012;31:388-95.

61. Stoltz JA, Shannon K, Kerr T, et al. Associations between childhood maltreatment and sex work in a cohort of drug-using youth. Soc Sci Med 2007;65:1214-21.

62. Argento E, Chettiar J, Nguyen P, et al. Prevalence and correlates of nonmedical prescription opioid use among a cohort of sex workers in Vancouver, Canada. Int J Drug Policy 2015;26:59-66.

63. Kerr T, Stoltz J-A, Marshall BDL, et al. Childhood trauma and injection drug use among high-risk youth. Journal of Adolescent Health 2009;45:300-2.

64. Ulibarri MD, Strathdee SA, Lozada R, et al. Intimate partner violence among female sex workers in two Mexico-U.S. Border cities: Partner characteristics and HIV risk behaviors as correlates of abuse. Psychological Trauma: Theory, Research, Practice, and Policy 2010;2:318-25.

65. Argento E, Strathdee SA, Goldenberg S, et al. Violence, trauma and living with HIV: Longitudinal predictors of initiating crystal methamphetamine injection among sex workers. Drug Alcohol Depend 2017;175:198-204

66. Tull MT, Gratz KL, Aklin WM, et al. A preliminary examination of the relationships between posttraumatic stress symptoms and crack/cocaine, heroin, and alcohol dependence. J Anxiety Disord 2010;24:55-62.

67. Romero-Daza N, Weeks M, Singer M. Conceptualizing the impact of indirect violence on HIV risk among women involved in street-level prostitution. Aggress Violent Behav 2005;10:153-70.

68. Wang B, Li X, Stanton B, et al. Sexual Coercion, HIV-Related Risk, and Mental Health Among Female Sex Workers in China. Health Care Women Int 2007;28:745-62.

69. Morgan CJ, Muetzelfeldt L, Muetzelfeldt M, et al. Harms associated with psychoactive substances: findings of the UK National Drug Survey. J Psychopharmacol 2010;24:147-53. 